\title{
Polymeric microelement on the top of the fiber formation and optical loss in this element analysis
}

\author{
Maria I. Fokina, Nina O. Sobeshuk, Igor Y. Denisyuk* \\ Saint-Petersburg State University of Informational Technologies, Mechanics and Optics, Saint-Petersburg, Russia; \\ *Corresponding Author: denisiuk@mail.ifmo.ru
}

Received 8 February 2010; revised 15 March 2010; accepted 21 March 2010.

\begin{abstract}
This paper describes the formation technology of polymeric microlenses self-organized on the fiber top in the limited reaction volume. The loss inserted to the optical coupling channel and their sources was investigated.
\end{abstract}

Keywords: Self-Focusing; Microlens; Optical Fiber; Photopolymer; Coupling

\section{INTRODUCTION}

Currently one of the important problems of fiber optics is the coupling of optical fibers with light sources and the introduction of light into the fiber with minimal losses. Direct welding of two fibers is optimal only for fibers with the same diameter and the aperture, while in other cases losses increasing require the use of focusing and collimating external systems. Loss of the light at fiber coupling whit LED laser or diode is too large, because emitting area, as the laser and the diode, always larger then diameter of fiber core. One solution to this problem is the use of focusing microelements on the top of the fiber.

A numerous effective technologies for microelements preparation, including microlenses made directly at the fiber top now are developed. Creating elements immediately at the fiber top is preferred because provides a selfalignment (coaxiality of axes) microlens and the fiber and no need for further manual assembly.

There are several well known methods for optical microelements preparation on the top of the fiber such as: laser fusion, or a gas burner (microoptical elements of various forms and purposes) [1], chemical etching (light coneconcentrator) [2], ion beam engraving (diffractive microlensese) [3]. The first two methods have found wide application, but the disadvantage is the possible misalignment of the microelement and core, and the de- fect of a second-the unavoidable chemical contamination of the surface. Technology of ion beam engraving allows to obtain micro-relief of very high quality and small size, but this method is technically difficult in expensive [3].

In recent years carried out many researches in the field of preparation of the polymeric optical microelements on the top of fiber [4-6]. The method is based on monomer composition polymerization deposited on the fiber top by laser radiation coming from the fiber. This technology allows obtaining high-quality microelements, as well as to control their size and profile [6]. However, all developments in this, of course, promising area, are at the stage of laboratory research, and experimental results depend from many different conditions such as type of monomer, initiator concentration, polymerization volume and differ in different works. In this work we prolong our experiments with microlens in fiber top self-writing made previously [7-9]. We find that processes of photopolymerization depend from speed of oxygen diffusion from monomers surface to polymerized area and so it depend from monomer volume. In this work we accomplish self-writing photopolymerization process in small monomer drop placed in fiber top in condition of fast oxygen diffusion from it surface. As oxygen will inhibit acrylate photopolymerization process [10].

\section{EXPERIMENT}

This work is devoted to investigation of the polymeric microelements formation processes at the end of optical fiber using laser radiation coming from the fiber at conditions of oxygen high speed diffusion to polymerization area. Figure 1 shows the scheme of "growing" elements.

Into the one end of the fiber 50/125 was introduced a $\mathrm{N} 2$ laser irradiation $(\lambda=337 \mathrm{~nm})$, on the other, vertically mounted end, a drop of the liquid monomer composition was deposited. The height of the drop was controlled 


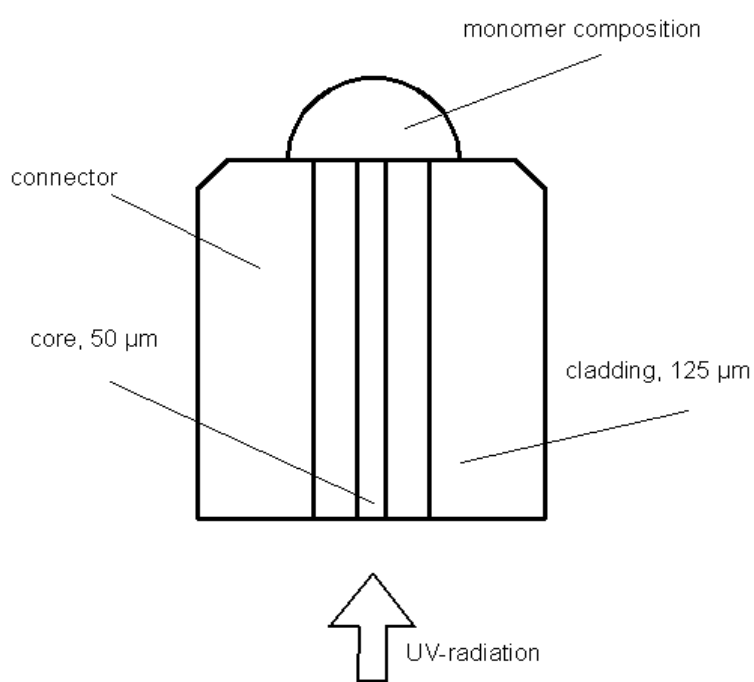

Figure 1. Scheme of the formation a polymeric element on the top of the fiber.

visually over microscope. A mixture of acrylates was used: 2-carboxyethyl acrylate (50\%), Bisphenol A glycerolate $(20 \%), 1,6$-hexanediol diacrylate $(10 \%)$ and Trimethylolpropane ethoxylate $(1 \mathrm{EO} / \mathrm{OH})$ methyl ether diacrylate $(20 \%)$. All reagents were purchased from Aldrich. As the initiator dimetoksifenilatsetofenon $(0.1 \%)$ with absorption maximum at $250 \mathrm{~nm}$ was used. At $337 \mathrm{~nm}$ its absorption length was few millimeters. The refractive index of the mixture is -1.48 . Exposure ranged from 3 up to 45 seconds ( $t=3,5,10,15,25,45 \mathrm{sec})$.

The elements formation process can be divided into following stages:

1) The process of polymerization doesn't begin immediately, but from the moment when the absorbed by monomer energy reaches a certain threshold. Delay appears because of the fact that the first impulses burn the oxygen in the monomer, which is the inhibitor of the process, after that only formation of free radicals from initiator molecules begin.

2) With a minimum exposure the formation of small, quasi-lens is observed, Figure 2.

This stage is the most difficult to reproduce because polymerization process takes place very rapidly and continuously.

3) Formation of cylindrical/conical shape element with rounded top, Figure 3.

4) The formation of the narrowing structure is possible (Figure 4).

5) There is an alignment of profile of the element while increasing exposure. Experiments have shown that this form (Figure 5) has a high repeatability (the probability of almost $100 \%$ ) when exposure element is sufficient.

After that visible light passing through microelements

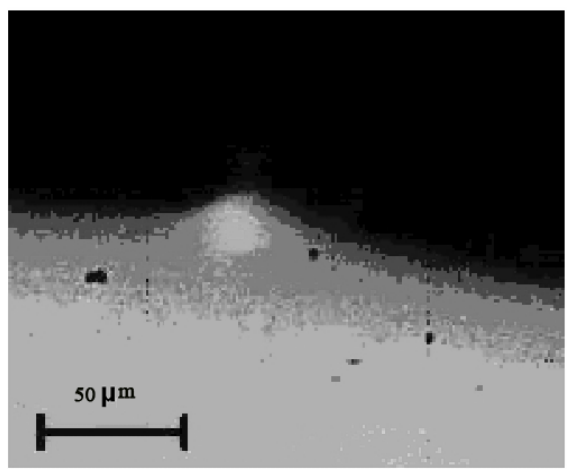

Figure 2. Element at the fiber top with the minimum exposure. Line of $50 \mu \mathrm{m}$ is shown in the photo.

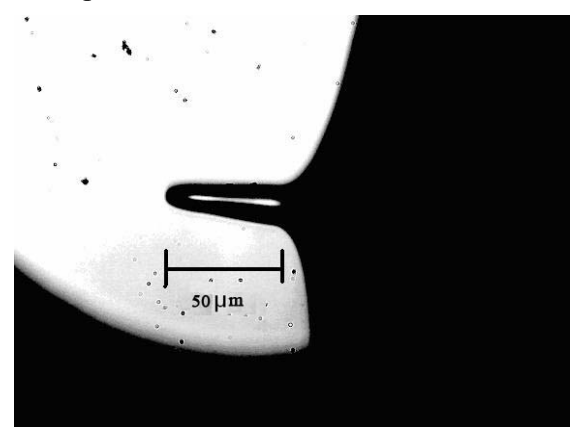

Figure 3. Polymeric element at the top of the fiber, exposure- $10 \mathrm{sec}$.

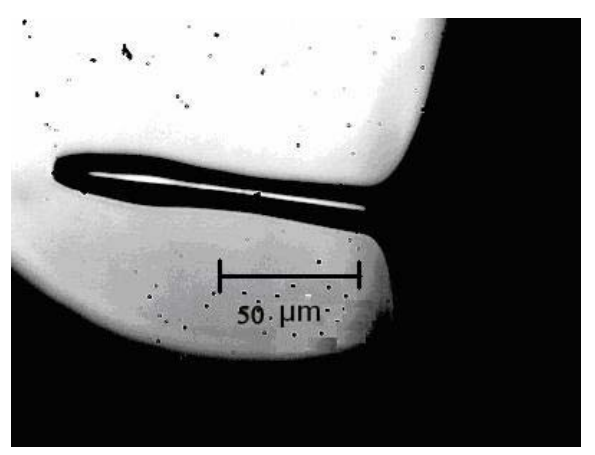

Figure 4. Polymeric element at the top of the fiber, exposure-15 sec.

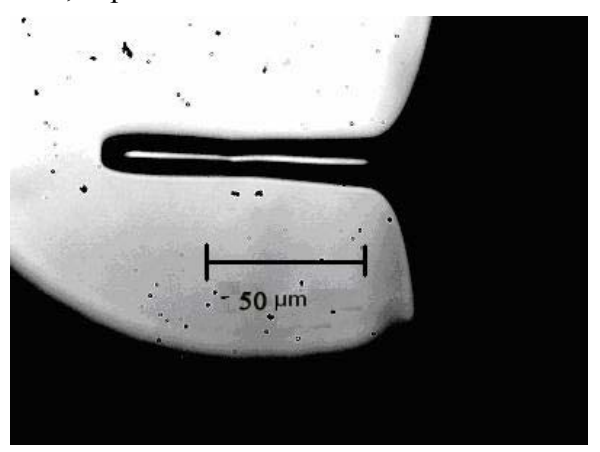

Figure 5. Polymeric element on the top of the fiber, exposure-45 sec. 
was investigated. The experimental scheme is shown on Figure 6.

The dependence of the radiation intensity on the distance between the fiber end without microelement and a scanning fiber connected to a photodetector was measured in the first part of the experiment. In the secondthe dependence of intensity on the distance between the scanning fiber and the fiber with polymeric microelement is on the end. As the scanning fiber $9 / 125 \mu \mathrm{m}$ was used, because most interesting is efficiency of using microelements in the coupling of fibers of different core diameters (single mode with multimode).

Figure 7 shows the results of measurements (dependence of the photodetector signal on the distance between the fibers). Line 1 corresponds to the first part of the experiment, the line 2-the second.

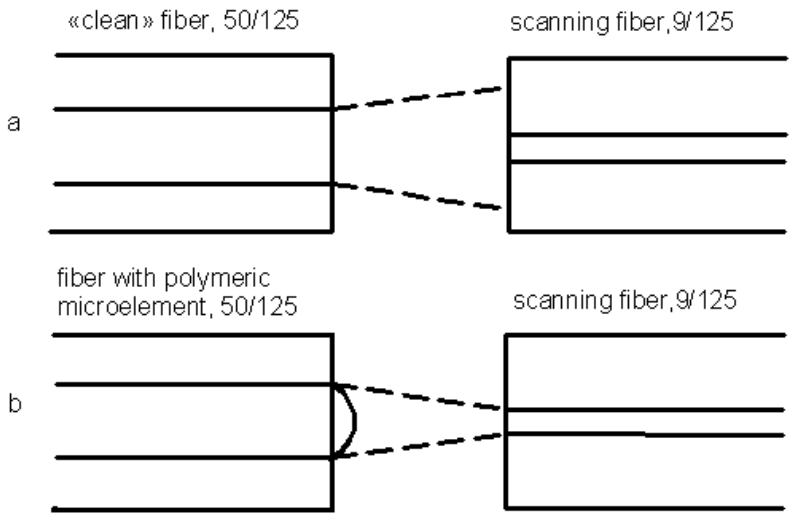

Figure 6. a) Measurement using fiber without microlens, b) Measurement using the fiber with polymeric microelement on the end.

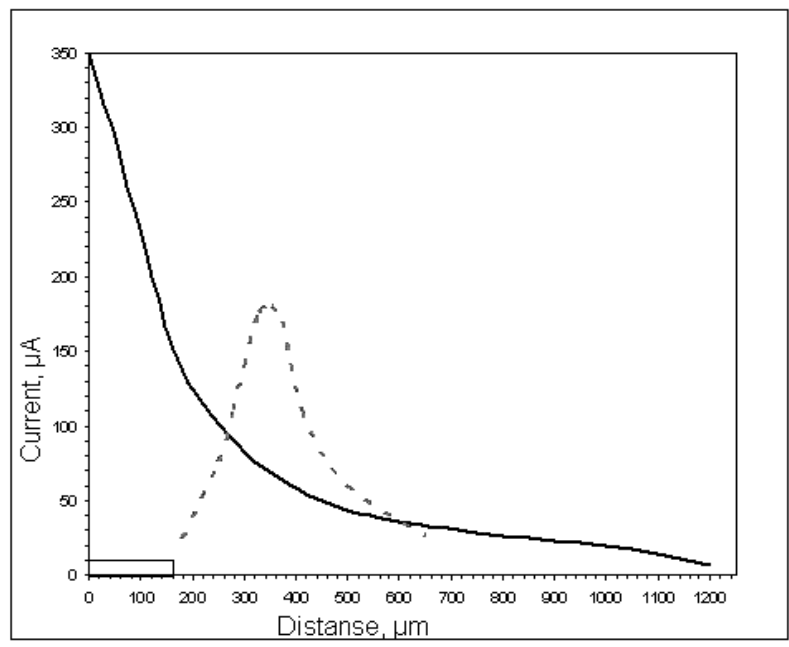

Figure 7. Dependence of the photodetector signal from the distance between the fibers: 1 (full line)-fiber without microlens; 2 (dashed line)-polymeric microelement on the end of the fiber.
Curve 2 has a clearly defined maximum, which confirms that the microelement focuses light. However, the graph shows that even at the maximum signal, consequently the intensity of radiation is lower than when fibers connected back to back. To clarify the source of the losses the following experiment was made: radiation of $\mathrm{He}-\mathrm{Ne}$ laser was introduced into the fiber with microlens at its end. Figure 8 shows the obtained result: element remains dark, but there are significant losses at the interface polymer-fiber.

\section{RESULTS DISCUSSION}

The dynamics of growth of self writing waveguide and formation of polymeric microelements at the end of the optical fiber was investigated in this work. Micro-photos made in series during formation of self-writing waveguide in the top of the fiber. The main stages of element formation are shown in Figure 9 (in the left-exposition time for each photo). One of the features of obtained samples is that the width of all elements, starting from the third formation phase, is approximately equal to the half-width of fiber core. This can be explained in the following way: after reaching the threshold energy

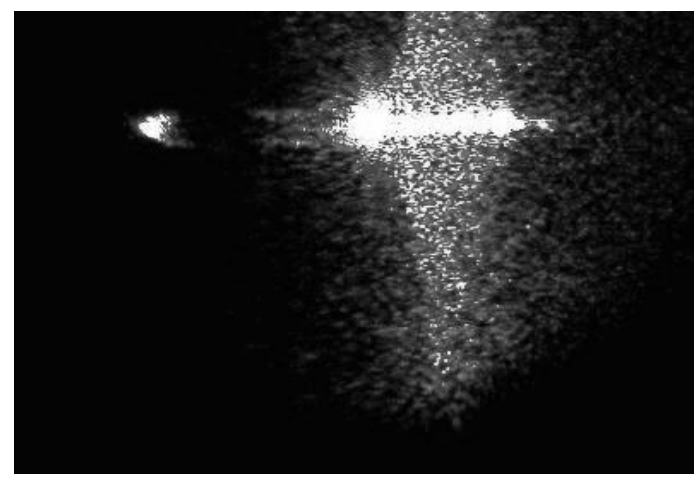

Figure 8. Passage of light through the fiber with a polymeric element at the end.

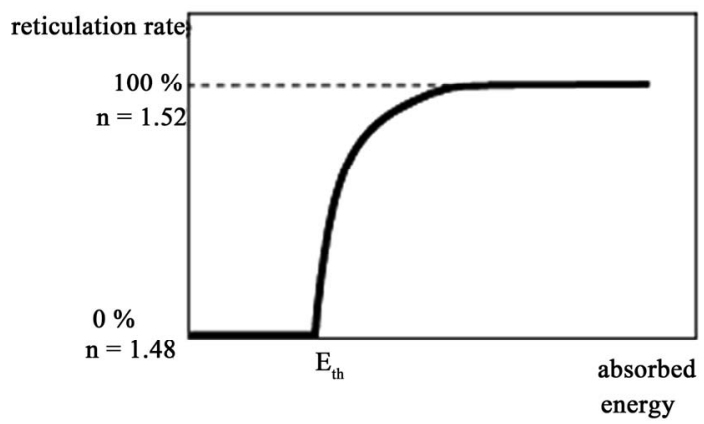

Figure 9. Empirical reticulation rate of the formulation versus absorbed energy. $E_{t h}$ is the threshold energy (achieved when the polymerization starts) and $n$ the refraction index of the formulation. 
the polymerization process takes place continuously and is accompanied by increasing of the refractive index Figure 9 [5].

For this composition increasing of the refractive indices is 0.04 (the monomer composition-1.48, the polymer-1.52). As a result, we see the effect of selffocusing of the light in the polymer, i.e. formation of the waveguide, the core of which is a polymer, and the cladding-unpolymerized monomer. It is known that even with full internal reflection light goes abroad the substance to the distance equal to half the wavelength, and, therefore, slow growth element in wide might be expected. However, this does not happen, because energy of emergent radiation is insufficient to burn oxygen and initiate the polymerization.

One of the components of monomer composition 2-carboxyethyl acrylate having a linear structure also provides directional growth of element from fiber glass surface. 2-carboxyethyl acrylate contains acid groups chemically active with quartz, which provide reaction of monomer with fiber top and beginning of element grown from fiber top away.

Also it was found that element length will stop in some moment and the radius of curvature of the drop increases when increasing exposure. Figure 10 shows photographs illustrating this effect.

We think that stopping of micro-element grown is a result of approaching of polymerized area to surface of monomer drop and, therefore, stopping of photopolymerization in result of oxygen inhibition influence.

Our investigation results showed that the fabrication method requires improving and optimization: significant losses in the microelement aren't observed, which confirms its waveguide structure, but there are significant losses at the interface between microelement and optical fiber. In fact is it the main component of optical losses and its removing will result on diminishing of optical losses to appropriate value.

Certainly, optical losses at interface: optical fiber/self writing waveguide resulting from narrowing of element in comparison to fiber core (fiber core $50 \mathrm{um}$; waveguide $25 \mathrm{um}$ ). Stepped transformation of waveguide core from 50 to 25 um will result on radiation going away at this interface.

In our opinion narrowing of polymerized area is a result of photo polymerization reaction inhibition by oxygen diffused from monomer drop volume to exposed area and slowing down of polymerization in surface of waveguide. Certainly this effect is negative and can be more powerful for single mode optical fiber having core diameter 7 um.

To overcome or reduce effect of waveguide narrowing according to our opinion are needed to diminish oxygen diffusion speed in monomer mixture, so are needed to

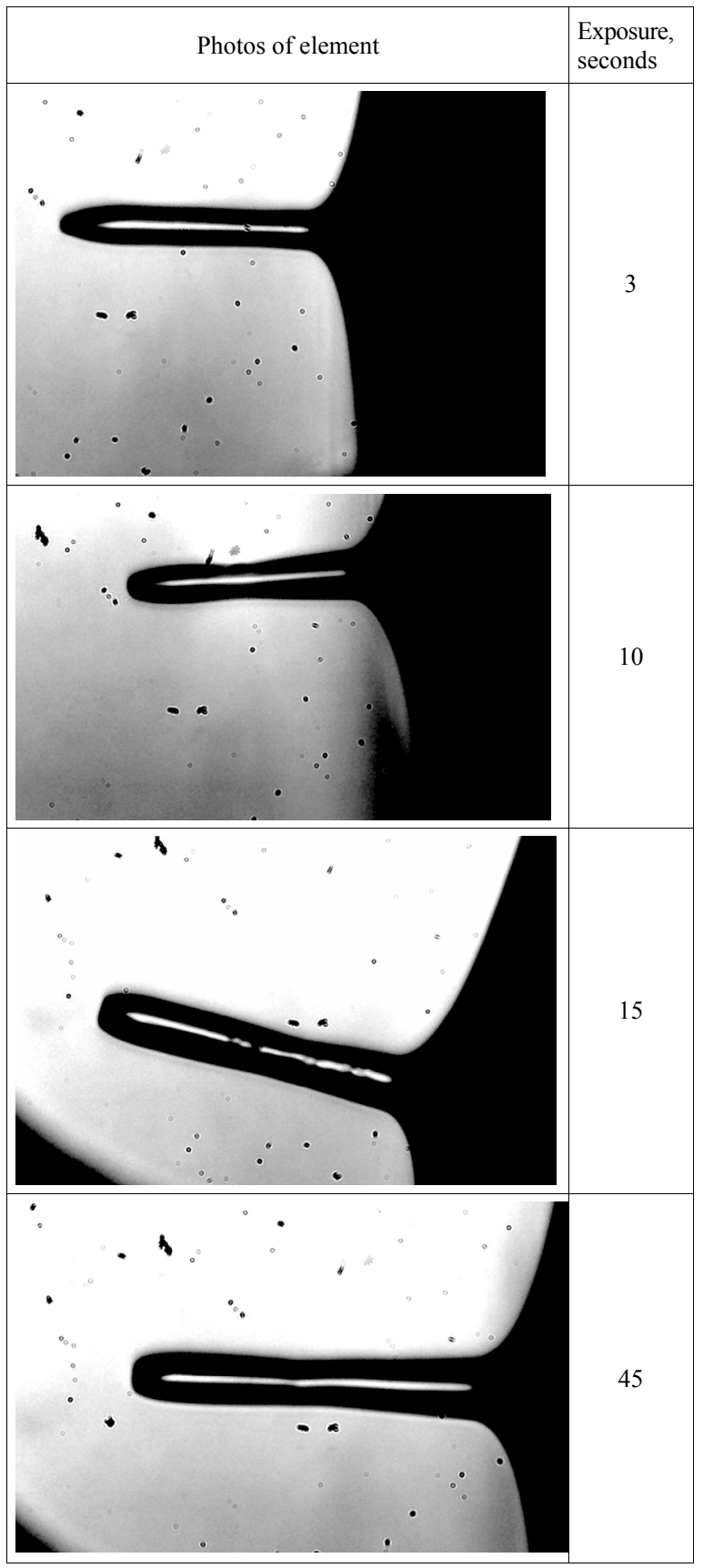

Figure 10. Dependence the radius of curvature of element from exposure.

change monomer composition to increase its viscosity or add traps for oxygen.

Now we prepare series of monomers composition with increased viscosity and with addition of nanoparticles as possible traps for oxygen diffusion. First experiments show improvement of waveguide formation.

Results obtained with these new UV-curable nanocomposite materials will be subject of next publication. 


\section{CONCLUSIONS}

In this work the processes of microelement self-writing by photopolymerization of composition drop deposited at the top of the optical fiber by laser radiation coming from the fiber are analyzed. Experimental investigation of focusing light by obtained microlenses was made, this study shows possibility of coupling microlens preparation with focal length of about $400 \mathrm{um}$.

It was shown that the observed loss of light in the microlenses formed by this method are determined by difference of diameters of self-organized light concentrator and fiber core, the losses directly in the microelement almost are not.

This work was made under support of Russian Ministry of Education grant RNP.2.1.1.3937.

\section{REFERENCES}

[1] Veiko, V.P., Berezin, S.D. and Chuiko, V.A. (1997) Laser technlogies for producing fiber-optical components. Bulletin of the Russian Academy of Sciences-Physics, 61(8), 1627-1631.

[2] Yang, Y., Lee, J., Reichard, K., Ruffin, P. and Liang, F. (2005) Fabrication and implementation of a multi-tosingle mode converter based on a tapered multimode fiber. Optic Communications, 249(1-3), 129-137.
[3] Schiapelli, A., Kumar, R., Prasciolu, M., Cojoc, D. and Cabrini, S. (2004) Efficient fiber-to-waveguide coupling by a lens on the end of the optical fiber fabricated by focused ion beam milling. Microelectronic Engineering, 73-74(1), 397-404.

[4] Hocinea, M., Bachelotb, R., Ecoffetc, C., Fressengeasa, N., Royerb, P. and Kugel, G. (2002) End-of-fiber polymer tip: Manufacturing and modeling. Syhthetic Metals, 127(1-3), 313-318.

[5] Barchelot, R., Ecoffet, C., Deloiel, D. and Royer, P. (2001) Integration of micrometer-sized polymer elements at the end of optical fibers by free-radical photopolymerization. Applied Optics, 40(32), 5860-5871.

[6] Plekhanov, A.I. and Shelkovnikov, V.V. (2006) Optical fibers with photopolymeric microlenses in the top. Nanotechnologies in Russia, 1(1-2), 240-244.

[7] Fokina, M. (2007) Optical surface making by UV-curing of monomeric compositions in near field of coherent light source. Molecular Crystals and Liquid Crystals, 468, 385-394.

[8] Fokina, M.I., Burunkova, J.E. and Denisuk, I.Y. (2007) Influence of photoactive additive on growth of polymer microelements on the top of optical fiber. SPIE Paper Number, 6732, 673215.

[9] Fokina, M.I., Kaporskiy, L.N. and Denisyuk, I.Y. (2008) Nature of microelements self writing in fiber tips in UV-Curable composites. Molecular Crystals and Liquid Crystals, 497, 236-240.

[10] Andrzejewska, E. (2001) Photopolymerization kinetics of multifunctional monomers. Progress in Polymer Science, 26(4), 605-665. 